\title{
El aprendizaje ubicuo en la educación superior: el dónde y cuándo del aprendizaje
}

\section{Ubiquitous learning in higher education: where and when of learning}

\author{
MSc, Oscar Riofrío-Orozco ${ }^{1}$ \\ oriofrio@utmachala.edu.ec \\ MSc, Ruth Moscoso-Parra \\ rmoscoso@utmachala.edu.ec \\ MSc, Javier Garzón-Montealegre \\ vgarzon@utmachala.edu.ec
}

Recibido: 1/12/2017, Aceptado: 1/02/2018

\begin{abstract}
RESUMEN
El presente artículo pretende explicar con rigor y claridad el estado del arte relacionado con el aprendizaje ubicuo. En este sentido y, en primer lugar, se analiza cómo el incremento de la actividad virtual telemática propicia el intercambio de datos, caracterizando al aprendizaje ubicuo como un nuevo paradigma educativo. Un segundo análisis, evidencia que el avance de las tecnologías obliga a desarrollar nuevas estrategias y modelos de contenidos digitales educativos. Un tercer análisis, pone de manifiesto el estado de la implementación del u-learning en la educación superior y analiza el rol del profesor frente al uso de los dispositivos digitales móviles.
\end{abstract}

Palabras clave: Aprendizaje ubicuo, ciudadanía móvil, movilidad, conectivismo

\section{ABSTRACT}

This article aims to explain with rigor and clarity the state of the art related to ubiquitous learning. In this sense and in the first place, it is analyzed how the increase of the virtual telematics activity propitiates the exchange of data, characterizing the ubiquitous learning as a new educational paradigm. A second analysis, evidence that the advance of technologies requires the development of new strategies and models of educational digital content. A third analysis reveals the state of the implementation of u-learning in higher education and analyzes the teacher's role in relation to the use of mobile digital devices.

Keywords: Ubiquitous learning, mobile citizenship, mobility, connectivity

1 Universidad Técnica de Machala 


\section{Introducción}

Hoy en día, cada vez más personas reconocen que los dispositivos digitales y la conectividad inalámbrica diseñan escenarios de formación con mejores oportunidades tanto para facilitadores como aprendices. El aprendizaje ubicuo es aquel que se realiza en cualquier momento y en cualquier lugar, potenciando el flujo de actividades y las relaciones diarias.

Esta sociedad caracterizada por la globalización del conocimiento, la instantaneidad, la interconectividad, se somete a cambios caracterizados por la gestión en el intercambio de información y plantea estrategias de intercambio de información y comunicativas más eficaces. Las tecnologías permiten crear nuevos soportes y nuevas formas para narrar el contenido.

Las instituciones de educación deben adaptarse a las nuevas demandas donde el incremento de la actividad virtual trae como consecuencia el intercambio oportuno de datos, de contenido. El aprendizaje ubicuo se convierte en un nuevo paradigma educativo. El incremento de nuevos servicios basados en audio y video permiten que la educación actual esté disponible en todo momento y en todo lugar.

El aprendizaje ubicuo genera para los profesores un recurso que permite conseguir las metas del aprendizaje más allá del aula de clase. Una cultura de aprendizaje ubicuo considera la actividad cotidiana como oportunidades para el aprendizaje. Aprender de las relaciones de red fomenta el tipo de razonamiento del cual depende la solución de problemas, el pensamiento crítico y la investigación.

\section{La Ubicuidad, transformando los contextos de formación}

Una de las principales características de la sociedad actual es la producción vertiginosa de conocimiento. Cualquier persona es productor y consumidor de información al mismo tiempo, hecho que no se encuentra limitado por condicionamientos físicos como espacios áulicos o bibliotecas, en este sentido las Tecnologías de la Información y Comunicación se convierten en herramientas potenciadoras a nivel mundial de cambios tanto a nivel institucional como personal.

La constante evolución tecnológica, la adopción de nuevas y mejores características de los dispositivos, la miniaturización y adaptabilidad del hardware, el abaratamiento de costos y, la portabilidad tecnológica permite la accesibilidad a los equipos dentro y fuera de las instituciones educativas.

La construcción del conocimiento a partir de las TIC hace dinámico el proceso de enseñanza-aprendizaje, motiva al trabajo colaborativo y cooperativo, crea amplios espacios de comunicación e investigación y sobre todo genera menos contenido fijo, aportando con la autonomía del proceso de aprendizaje.

Según Burbules (2012), cada vez más personas reconocen que los dispositivos móviles y la persistente conectividad inalámbrica promueven oportunidades de aprendizaje a más gente, en más contextos con costo mínimo o de forma gratuita. Esta idea de aprendizaje ubicuo significa que el aprendizaje se transforma en una proposición de cualquier momento y en cualquier lugar. Para Vázquez y Sevillano (2015), la construcción del conocimiento, a partir de las tecnologías impregna a las personas de mayor motivación e interés por el aprendizaje. 


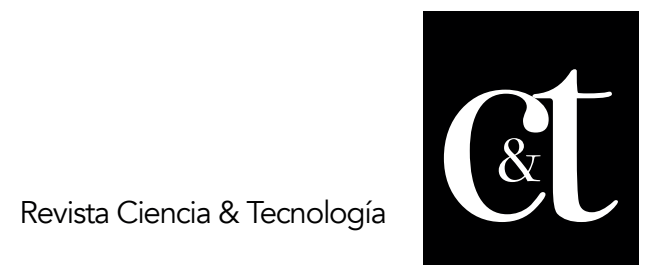

No. 18,30 de abril de 2018

ISSN impreso: 1390 - 6321

El aprendizaje ubicuo hace referencia al desarrollado en cualquier momento o lugar a través de dispositivos móviles. El significado de la palabra ubicuo es sinónimo de omnipresente, estar en todas partes. En cualquier lugar del mundo se puede acceder/obtener y difundir información gracias a la evolución tecnológica, sin olvidar la capacidad de evolución humana (Vázquez y Sevillano, 2015, p.22).

El aprendizaje ubicuo puede ser concebido como una alternativa para la formación complementaria, en la que los profesores ofrecen contenidos en horarios no lectivos, facilitando el desarrollo de actividades extracurriculares educativas, creando un escenario educativo más dinámico en la que los actores del proceso intercambien colaborativamente información. A este nuevo paradigma, se asocian otros elementos como el m-learning, aprendizaje a través de dispositivos móviles, celulares, i-Pod, SmarthPhone, Tablets y en general todo dispositivo móvil que disponga de conectividad inalámbrica. Se define el ulearning como la formación accesible en distintos canales al mismo tiempo y en cualquier lugar.

A partir del aprendizaje m-learning o móvil, las instituciones de educación deben reconsiderar las metodologías de enseñanza, el rol del docente y sobre todo considerar al alumno como participantes creativos y comunicativos. En estos contextos en donde prima el conocimiento global, es necesaria una transformación en los protocolos de comunicación. Las tecnologías ofrecen dos vertientes complementarias: son nuevos soportes formales y nuevas formas de narrar contenido (Fombona, 2008). La educación debe adaptarse a estos requerimientos en donde el intercambio de datos se realiza con un fuerte contenido narrativo audiovisual.

La rápida evolución de la tecnología informática transforma los procesos actuales, caracterizando a la educación por un fuerte paradigma colaborativo y conectivo que requiere profundos cambios, desde una nueva perspectiva del estudiante con nuevas competencias por adquirir hasta la necesidad de implementar nuevas formas de movilidad a fin de evidenciar una educación global y no delimitada por fronteras. Se debe preparar a los alumnos para un futuro digital.

El futuro de la formación docente tendrá que abordar el aprendizaje ubicuo: la posibilidad de acceder a la información en cualquier lugar o cualquier momento, la interacción con pares y expertos eruditos y oportunidades estructuradas de aprendizaje desde una variedad de fuentes. La brecha entre el aprendizaje formal e informal desaparecerá. A menudo este aprendizaje será "ajustado al tiempo", anclado a las necesidades de una cuestión, un problema o una situación inmediata (Burbules, 2012).

Actualmente no es posible concebir que el alumno aborde conocimiento desde una fuente autorizada, en la sociedad de la información estos crean el conocimiento de una forma activa. La utilización de las tecnologías como medio para descubrir el mundo que nos rodea confluye en un conocimiento ubicuo cuyas implicaciones en el aprendizaje formal no pueden negarse (Cope y Kalantzis, 2009).

El aprendizaje ubicuo es un nuevo paradigma educativo, que surge con la aparición de medios tecnológicos que permiten la interacción y acceso a contenidos. En este sentido, el alumno tiene la posibilidad de ampliar el abanico de posibilidades de 
interacción con profesores, con compañeros, acceder a información y contenidos de interés, valorar el aporte de otros a temas que considere de interés, explorar en alternativas tecnológicas para solucionar problemas.

\begin{tabular}{|l|l|}
\hline Permanencia & $\begin{array}{c}\text { - No existe perdida de información y el } \\
\text { proceso de aprendiaje es recordado } \\
\text { Et acceso a contenido en distinto } \\
\text { formato no esta limitado por tiempo ni } \\
\text { espacio } \\
\text { Acceso al contenido en el momento en } \\
\text { que consideren necesario }\end{array}$ \\
\hline Inmediatez & $\begin{array}{l}\text { Se interactua con la tecnología misma } \\
\text { y con profesores, compañeros, etc }\end{array}$ \\
\hline Interactividad & $\begin{array}{l}\text { Los problemas y el conocimiento estan } \\
\text { presente de forma natural y auténtica }\end{array}$ \\
\hline Actividades situadas & $\begin{array}{l}\text { Información correcta, en el lugar y } \\
\text { tiempo correcto }\end{array}$ \\
\hline Adaptabilidad & \\
\hline
\end{tabular}

Figura 1. Características del aprendizaje ubicuo

Fuente: Elaborado a partir de los aportes de McLean (2003), Houser \& Thornton (2004), Yu Liang (2005)

A fin de garantizar entornos de formación ubicuo, se deben considerar teorías educativas que consideren la tecnología como, por ejemplo, la cognición distribuida integrada en el campo de la interacción humano-computadora (HCI) la misma que opera, en su sentido más amplio, con la idea de compartir información y construir conocimiento.

La educación superior se caracteriza por la innovación permanente, y en este sentido se deben preparar a los alumnos y profesores para un escenario digital en el que se combinan las clases magistrales tradicionales con cursos, talleres, grupos colaborativos internacionales, combinación soportada por la tecnología que permite estar en distintos lugares al mismo tiempo.

Aprendizaje ubicuo, desde la perspectiva pedagógica

Tradicionalmente se concibe al aprendizaje como un proceso lineal en el que el sujeto recibe información confirmada desde una sola fuente. En la sociedad de la información y del conocimiento los aprendices crean de forma activa el conocimiento. Desde la segunda década del siglo XXI, los principios del proceso de enseñanza aprendizaje se basan en la conectividad. Los conceptos de comunicación democrática, participación, colaboración o empoderamiento se han extendido y tratan de apartar al paradigma transmisor y lineal que imponía las relaciones entre docentes y estudiantes (Aparici, 2011).

Para Siemens (2008) el conectivismo pondera la importancia de los dispositivos móviles como recurso para realizar actividades cotidianas, siendo la tecnología el elemento central en nuestra distribución de identidad, la cognición y el conocimiento. Como se aprecia, el conectivismo es la teoría principal donde se sustenta el aprendizaje ubicuo, del cual se desprende la definición como aquel aprendizaje 


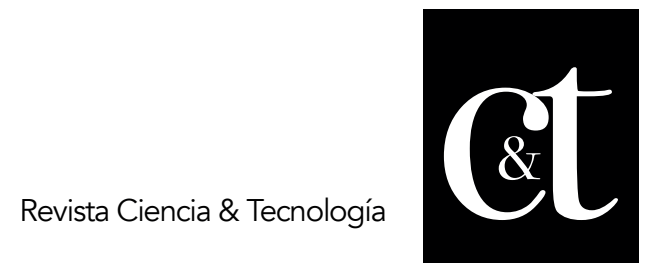

No. 18, 30 de abril de 2018

ISSN impreso: 1390 - 6321

caracterizado por el uso de tecnología permitiendo que la educación esté disponible en todo momento, en cualquier lugar y en cualquier medio social gracias a los dispositivos móviles.

En el conectivismo, el conocimiento es concebido como un conjunto de relaciones, y el aprendizaje como la creación de nuevas conexiones. Desde este punto de vista y con la facilidad que presta actualmente la tecnología digital, la creación de redes se consigue de forma fácil constituyéndose en elementos importantes en distintos aspectos de la vida. El conectivismo entiende el carácter cambiante del conocimiento, prevaleciendo las conexiones con los demás y su contexto (Siemens, 2008).

El avance de las tecnologías obliga a replantear nuevas estrategias y contenidos digitales educativos. Las características del u-learning o aprendizaje ubicuo propicia la integración de modelos de conocimiento y de contenido informativo (Rodrigo y Castro, 2013). La ventaja del aprendizaje ubicuo sobre otros modelos pedagógicos reside en su alto nivel de movilidad e integración, convirtiendo al proceso de enseñanza aprendizaje en omnipresente y en el que el estudiante avanza de forma autónoma, con una participación más activa.

La intromisión de los dispositivos móviles en el ámbito educativo debe conllevar a que las prácticas comunicativas lineales y unidireccionales, emisor-receptor, se conviertan en un medio de comunicación masivo, que permitan una transformación hacia un nuevo paradigma de aprendizaje colaborativo, creativo, horizontal y bidireccional, anulando la jerarquía del poder y dominio de unos sobre otros (Vázquez y Sevillano, 2015, p.43).

En la actualidad se observa una convergencia o complementariedad entre el aprendizaje tradicional y el ubicuo. Downes (2008) no habla de una sustitución de unos aprendizajes sobre otros, sino de una convergencia gradual entre las diferentes formas de aprender. Sevillano, Vázquez y Ortega (2013) señalan que se está produciendo una revolución en los contenidos, recursos y espacios tradicionales de enseñanza aprendizaje y que en ellos se destacan aspectos como discusión, colaboración, exploración y reflexión.

Los dispositivos móviles incorporan múltiples aplicaciones ofreciendo posibilidades en el ámbito educativo. Rodrigo y Castro (2013), enfatizan en la posibilidad de estos dispositivos creando escenarios de formación más accesibles, utilizables y adaptables a todos los usuarios, facilitando la comunicación de asistencia y aumento de la movilidad.

\section{Los dispositivos digitales móviles en la educación superior}

Se entiende por dispositivo a un mecanismo o artefacto útil para realizar determinada acción para la que ha sido diseñado (RAE, 2014). Atendiendo al modo que tienen para procesar la información, los dispositivos pueden ser analógicos y digitales. Los primeros realizan mecánicamente la acción, los digitales lo hacen electrónicamente. Bajo esta clasificación, se puede ejemplificar a una máquina de escribir como dispositivo analógico y a una computadora portátil como dispositivo digital.

Los dispositivos digitales pueden ser fijos y móviles. Los fijos realizan la acción para la cual fueron creados si se mantienen con suministro eléctrico, sin alimentación 
eléctrica permanecen inoperantes, en cambio los dispositivos digitales móviles han sido diseñados con independencia del suministro eléctrico por cable. Así, una computadora de escritorio es un dispositivo digital fijo y una computadora portátil es un dispositivo digital móvil.

En el mundo de la formación superior, la universalidad en el uso de los dispositivos digitales móviles no ha sido conseguida todavía. En esta dirección se están implementando algunas estrategias que permitan disminuir esta característica mediante el uso de libros tradicionales y ebooks, el uso de computadoras portátiles, acceso a información desde teléfonos celulares. En algunos casos las pizarras digitales conviven ya con las pizarras de tiza o acrílicas.

El poco uso de tecnología digital en la educación superior está dando sus frutos. Los nuevos recursos didácticos están desarrollando en los estudiantes otro tipo de competencia, la tecnológica o digital, desarrollando un perfil estudiantil caracterizado por la autonomía, mayor grado de interacción, una toma de decisiones más estilizada y una mayor colaboración y comunicación.

En la educación superior converge una población heterogénea de estudiantes, caracterizada principalmente por el grado en el que han incorporado en su actividad formativa metodologías tradicionales o de uso de TIC, aplicando esta caracterización también al claustro docente, considerando principalmente la edad promedio de los profesores. En el claustro docente predominan los inmigrantes digitales sobre los nativos digitales. Este hecho puede crear en las aulas una natural brecha digital generacional que unida a la brecha digital formativa (Quicios, Ortega y Trillo, 2014) facilitan que en la educación superior coexistan múltiples metodologías digitales.

Los profesores utilizan el e-learning en cualquiera de las tres generaciones (Gros \& García-Peñalvo, 2016; Cabero, 2013):

- Primera generación, adaptación de material textual a formato web

- Segunda generación, se enfatiza en la búsqueda del mejor campus virtual

- Tercera generación, trata de que el estudiante genere el conocimiento de forma conjunta, apoyándose en la flexibilidad y colaboración. Considera la superación de la metáfora del aula, accediendo al contenido a través de distintos dispositivos.

Sea cual fuere la generación adoptada por el profesor en educación superior, la mayoría la desarrolla apoyándose de computadoras portátiles, constituyéndose en el primer dispositivo digital móvil utilizado para fines formativos. La potencialidad de este dispositivo radica en el software que lo acompaña, así podrá ser utilizado como la antigua máquina de escribir, si explota únicamente un procesador de texto o apoyándose en las distintas versiones de la web, cada una de las cuales representa una velocidad formativa diferente. Para Vázquez y Sevillano (2015), la velocidad digital formativa elegida por el profesor depende de aspectos como, su pericia tecnológica, la capacidad tecnológica del centro educativo, el software que disponga, la naturaleza de la asignatura que imparte, la capacidad de los estudiantes. Para estos autores la velocidad digital formativa elegida por el profesor determinará su rol, así:

- Web1.0, el docente seguirá siendo el especialista que transmite 


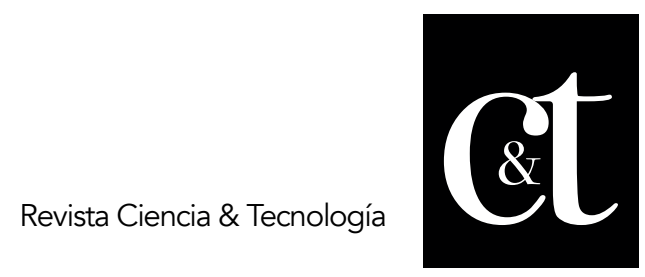

No. 18, 30 de abril de 2018

ISSN impreso: 1390 - 6321

conocimientos.

- Web 2.0, el docente adopta el papel de guía que facilite el escenario al estudiante para que descubra el conocimiento.

- Web 3.0, el docente se constituye en una pieza más del puzzle formativo.

La clave de la calidad formativa a través de dispositivos digitales móviles radica en la posibilidad del uso del software existente en el mercado, más que en la potencialidad del hardware de los dispositivos.

\section{Conclusiones}

El mundo está cambiando a gran velocidad, pasando de una economía industrial a una economía basada en la información y el conocimiento que surge apoyada por medios tecnológicos. A medida que la sociedad se hace más pequeña por la aparición de los medios de comunicación nuestra capacidad para ser consumidores, pero también productores de información aumentan.

El potencial generado por el desarrollo tecnológico en escenarios de la información y comunicación posibilita que toda persona pueda producir y diseminar información en cualquier momento y en cualquier lugar. Esta característica, conocida como ubicua permite usar la tecnología rompiendo las barreras espacio temporales, confluyendo lo físico con lo virtual, movilizando los dispositivos de oficinas, aulas o espacios cerrados a espacios sociales y públicos.

El aprendizaje ubicuo funde el ahora y el cuándo. Nos brinda la posibilidad de comunicación asíncrona, de tal manera que no hace falta que una clase empiece a una hora determinada. Ahora puede ser antes o después.

\section{Referencias bibliográficas}

Aparici, R. (2011). Principios pedagógicos y comunicacionales de la educación 2.0. Organización de los Estados Iberoamericanos. 145. Recuperado de http://www.educoas.org/portal/La_Educacion_Digital/laeducacion_145/articles/ Roberto_Aparici.pdf

Burbules, N. C. (2012). El aprendizaje ubicuo y el futuro de la enseñanza. Encounters on education = Encuentros sobre educación = Recontres sur l'éducation, (13), 314

Cabero, J. (2013). El aprendizaje autorregulado como marco teórico para la aplicación educativa de las comunidades virtuales y los entornos personales de aprendizaje. Revista Teoría de la Educación: Educación y Cultura en la Sociedad de la Información, 14(2), 133-156. Recuperado de http://tecnologiaedu.us.es/tecnoedu/images/stories/jca101.pdf

Cope, B. y Kalantzis, M. (2009). Ubiquitous Learning. Exploring the anywhere/anytime posibilities for learning in the age of digital media. University of Illinois Press. Recuperado de http://www.nodosele.com/blog/wpcontent/uploads/2010/03/Cope Kalantzis.Ap rendizaje ubicuo.pdf

Downes, S. (2008). El futuro del aprendizaje en línea: Diez Años Después. Recuperado de http//es.scribd.com/doc/16527898/El-Futuro-del-Aprendizajeen-Linea-Diez-años-Después 
Fombona, J. (2008). Lectura de imágenes y contenidos. Competencias para el análisis de la forma y contenidos del audiovisual: Hacia una teoría de la composición. Madrid: CEP

Gros, B. \& García-Peñalvo, F. J. (2016). Future trends in the design strategies and technological affordances of e-learning. Learning, Design, and Technology: An International Compendium of Theory, Research, Practice, and Policy, 1-23.

Houser, C, \& Thornton, P. (2004). Japanese college students typing speed on mobile devices. Actas del 2nd IEEE International Workshop on Wireless and Mobile Technologies in Education, 24-25. Recuperado de http://reusability.org/read/chapters/wiley.doc

Quicios, M., Ortega, I., y Trillo, M. (2014). Aprendizaje ubicuo de los nuevos aprendices y brecha digital formativa. Pixel-Bit: Revista de medios y educación, $46,155-166$

McLean, N. (2003). The M-Learning Paradigma Overview. Informe para la Royal Academy of Engineering and the Vodafone Group Foundation, Sydney. Recuperado de http://www.oucs.ox.ac.uk

RAE (2014). Diccionario de la Lengua Española. Recuperado de http://www.rae.es/recursos/diccionarios/drae

Rodrigo, M. y Castro, C. (2013). La información digital actual, un nuevo modelo de contenido educativo para un entorno de aprendizaje ubicuo. Revista de Educación a Distancia RED, $39 . \quad$ Recuperado de http://www.um.es/red/39/AlonsodeCastro.pdf

Sevillano, M., Vázquez, E., y Ortega, J. (2013). Herramientas virtuales ubicuas y móviles en la innovación formativa: tabletas y ordenadores portátiles. Textos. Revista internacional de aprendizaje y cibersociedad. 17, (2), 71-87

Siemens, G. (2008). Conectivismo: una teoría del aprendizaje para la era digital. Recuperado

de http://humanismoyconectividad.wordpress.com/2009/01/14/conectivismosiems L

Vázquez, E., y Sevillano, M. (2015). Dispositivos digitales móviles en educación: El aprendizaje ubicuo. Madrid: Narcea.

Yu-Liang, R. (2005). Mobile Learning-Current Trend and Future Challenges. Actas del Fifth IEEE ICALT05. Taiwan: Kaohsiung. 